\title{
Spectrum of acute hepatitis B at tertiary care center of northern India
}

\begin{abstract}
Introduction: Hepatitis is often a viral infection and hepatitis B virus (HBV) is reason in significant proportion of cases worldwide. HBV impact on liver can be of varying severity ranging from acute hepatitis to chronic hepatitis which can further lead to chronic liver disease, liver cirrhosis and hepatocellular carcinoma (HCC). There is paucity of data on acute hepatitis B, thus more researches will lead to better understanding of epidemiology and planning appropriate strategies required for prevention and control.
\end{abstract}

Aims and objectives: The aim was to study Clinico-epidemiological profile in patients having acute infection of HBV and to assess the various risk factors, age \& sex distribution and geographic foci of the disease, if any.

Materials \& methods: It was an epidemiology based, prospective study conducted at Medical Gastroenterology Department,PGIMS,Rohtak over a period of three years . Four hundred and nine (409) patients who were found to be having features of acute hepatitis and confirmed to be positive for HbsAg on Enzyme linked Immunoassay test (ELISA) and HBV DNA on PCR testing were enrolled in the study.

Results: The burden of acute hepatitis B infection is more in the young males especially in the rural areas i.e. residing in villages. The reason for this could be lack of awareness, hygiene and shortage of health facilities.
Volume 12 Issue I - 202 I

\author{
Parveen Malhotra, 'Vani Malhotra, ${ }^{2}$ Usha \\ Gupta, ${ }^{3}$ Gill PS, ${ }^{4}$ Pushkar, ${ }^{4}$ Yogesh Sanwariya ${ }^{5}$ \\ 'Department of Medical Gastroenterology and Microbiology, \\ Post Graduate Institute of Medical Science, India \\ ${ }^{2}$ Department of Obstetrics \& Gynecology, Post Graduate \\ Institute of Medical Science, India \\ ${ }^{3}$ Director, Health Services, Post Graduate Institute of Medical \\ Science, India \\ ${ }^{4}$ Department of Microbiology, Post Graduate Institute of \\ Medical Science, India \\ ${ }^{5}$ Department of Medical Gastroenterology, Post Graduate \\ Institute of Medical Science, India
}

\begin{abstract}
Correspondence: Parveen Malhotra, Department of Medica Gastroenterology and Microbiology, Post Graduate Institute of Medical Science, India, Tel 91925372663.

Email drparveenmalhotra@yahoo.com
\end{abstract}

Received: December 23, 2020 | Published: January 27, 2021

Keywords: hepatitis B, acute hepatitis B, HbsAg, tattooing, blood transfusion

Abbreviations: HbsAg, hepatitis B surface Antigen; ALT, alanine transferase; ELISA, enzyme linked immunoassay; PCR, chain reaction; $\mathrm{CI}$, confidence intervals

\section{Introduction}

HBV infection is one of the major health problems being faced by India and its large population is cause for increased burden of the same. The Hepatitis B surface Antigen ( $\mathrm{HbsAg}$ ) positivity in India is variable in different states and ranges between $2-4.7 \% .^{1,2}$ It is expected that around 40million people are chronically infected with Hepatitis B in India ${ }^{3}$ but there are no large scale studies on acute hepatitis B. The people at risk are who receive multiple blood/ blood products transfusion, chronic renal failure patients on maintenance hemodialysis, intravenous drug abusers, males having sex with male, female sex workers, sexual partners and family members of infected people, prisoners etc. Hepatitis B over a prolonged period may progresses to cirrhosis of liver and hepatocellular carcinoma, thus mandates early intervention for decreasing morbidity and mortality associated with it. ${ }^{4}$ Acute hepatitis B can be prevented by easily and widely available vaccine. The hepatitis $\mathrm{B}$ vaccination of newborns and high risk group is showing good results in most countries. ${ }^{5}$ The majority of Hepatitis B infection are asymptomatic and may present only with mild elevation of transaminases. Many trials related to vaccine has shown that many patients developing hepatitis B infection will have some liver enzyme elevation and after an incubation of 2-24weeks, jaundice develops in about $14-30 \%$ of infected individuals..$^{6-8}$ The development of symptomatic disease following $\mathrm{HBV}$ infection is determined by the infectious dose within an inoculums, ${ }^{9}$ the pathogenicity of the infecting strain, and the immune response of the host. There are very sparse studies available on acute hepatitis $\mathrm{B}$ infection which is needed for understanding its epidemiology and which may help in the disease prevention and control. Hence, the present study was undertaken to determine epidemiological profile of acute hepatitis B infection.

\section{Aim and objectives}

The aim was to study Epidemiological factors in patients having acute infection of hepatitis B virus and to determine the risk factors, age, sex and geographical distribution.

\section{Materials and methods}

The present study was a hospital based, prospective, observational study conducted at PGIMS, Rohtak for three years i.e. Ist April 2017 to 31st March 2020, for determining epidemiological factors related to acute hepatitis B infection. An informed consent was obtained from each patient prior to enrollment into the study. A detailed history and physical examination was carried out and samples were collected for every subject. The details of patients recorded included age, sex, rural or urban location, occupation, risk factors exposure especially in last six months like history of blood transfusion, tattooing, surgical or dental intervention, any hospitalization requiring injections or intravenous fluid requirement, intravenous drug abuse, history of jaundice in other family members or nighbourhood. An AVH case was defined as a person having an acute illness of $<15$ days duration with a discrete onset of any sign/symptom (e.g. Fever, headache, malaise, anorexia, nausea, vomiting, diarrhea, and abdominal pain) and either a) jaundice or b) elevated serum alanine transferase (ALT) $>100 \mathrm{IU} / \mathrm{L}$ documented atleast twice at a 1week interval without any history of pre-existing liver disease. ${ }^{10}$ The patients having co-infection with hepatitis C \& HIV, alcoholic, autoimmune, hemolytic, malarial, drug induced hepatitis, cholestatic jaundice, chronic liver disease or acute or chronic liver disease were not included in the study. Around 7-10ml 
of blood was collected from all the patients. Serum was separated by centrifugation, aliquoted \& stored at -20 degree till further tests were performed. Every subject underwent detailed set of investigations which included like complete haemogram, liver \& renal function test, ESR,INR, anti-HAV IgM ELISA, HbsAg ELISA, anti- HCV IgM ELISA, anti HEV IgM ELISA assay, and ultrasound abdomen.

Four hundred and nine (409) patients who were found to be having features of acute hepatitis and confirmed to be positive for HbsAg on Enzyme linked Immunoassay test (ELISA) and HBV DNA by polymerase chain reaction (P.C.R) testing and were enrolled in the study.

\section{Analysis of data}

All the relevant collected data was analyzed using SPSS (Statistical Package for Social Studies) and for differences between groups, Pearson's chi-square test was used. For calculating difference of means for quantitative variables, "t" test was used. The distributed data were presented as means and standard deviation, or $95 \%$ confidence intervals (CI), the $\mathrm{p}$ value of less than 0.05 was considered significant.

\section{Observations and results}

The present study analyzed the data pertaining to 409 patients and had male predominance i.e. $300(73 \%)$ in comparison to females i.e. 109 patients $(27 \%)$. There was rural predominance i.e. $343(83 \%)$ of the subjects came from rural areas (village background) and only $68(17 \%)$ were having urban background. Out of total 409 patients, eighty four percent patients were married. The age group varied from $7 \mathrm{yrs}-80$ yrs of age but maximum number of patients belonged to 20 to 50 years age i.e. $66 \%$ of total patients. At extreme of ages i.e. from 0 yrs-10 yrs \& $70 \mathrm{yrs}-80 \mathrm{yrs}$, only $3(1 \%)$ patients were found to be having acute hepatitis B. More than one third i.e. 150 patients (\%) were from Rohtak district alone but it could not be inferred that this district is having highest prevalence but the institute where we conducted this study is located here, thus due to easy accessibility, majority of patients came for treatment. The most striking thing noticed on data analysis was that there are certain geographical hotspots i.e. districts like Sonepat (41 i.e. 10.02\%), Jind (36 i.e. 8.80\%), Karnal (30 i.e.7.33\%), Panipat (26 i.e. 6.35\%), Kaithal (20 i.e. 4.88\%) showed significant proportion of cases of acute hepatitis B and they are already having high prevalence of hepatitis C. ${ }^{11}$

In present study, history of dental procedures (96 patients, $23.47 \%$ ), previous surgery (71 patients, $17.35 \%$ ) and tattooing (52 patients, $12.71 \%$ ) appeared as major risk factors. The history of intake of alternative medications was found in $51(12.46 \%)$ patients and 70 (17.11\%) patients had history of alcohol intake. Chronic Hepatitis C and Human immunodeficiency virus (HIV) was found in $2(00.48 \%)$ $\& 5(1.22 \%)$ patients respectively. There were no risk factors found in $62(15.15 \%)$ patients. Surprisingly no patient admitted for intravenous drugs abuse which can be due to willful hiding of information for personal reasons (Figures1-6).

\section{Discussion}

HBV infection endemicity has been reported in various countries but now more number of cases is being diagnosed to be suffering from HBV infection and related morbidity \& mortality. Around 2 billion people have been infected worldwide and out of them, 350 million suffer from chronic HBV infection. In $20-30 \%$ of adults who are chronically infected will develop cirrhosis and/or liver cancer. ${ }^{12-15}$ The prevalence of Hepatitis B surface antigen (HBsAg) is used to classify geographical areas as high (where $>8 \%$ of the population is $\mathrm{HBsAg}$ positive), intermediate $(2-7 \%)$ or low $(<2 \%) \mathrm{HBV}$ endemicity. ${ }^{16}$ One of the most important reasons for diagnosing of more number of cases of acute hepatitis B is more frequent testing of complete viral screen i.e. HbsAg, anti HCV antibody and anti HIV antibody test in patients of acute viral hepatitis which was not done in past practice of dealing with such patients. The reasons for diagnosing more cases of acute hepatitis B can be attributed to increase testing for HbsAg in jaundice patients. Moreover other important factors for detecting more patients of acute hepatitis B is due to HbsAg testing before surgery as Preanesthetic check up, in pregnant females, in voluntary blood donors and screening camps for Hepatitis B \& C. However, increased number of acute hepatitis B patients can also be attributed to widespread availability of injectable therapies, that too without proper safety precautions and the illicit use of injectable drugs. The present study highlights more involvement of male gender in younger age group. The reason for very low percentage of cases in below ten years of age group can be due to effective implementation of hepatitis B National immunization program under which for last four years, majority of the newborns born in India are started on hepatitis B vaccination at the time of birth. The lesser involvement of older age group i.e. above $70 \mathrm{yrs}$, can be attributed to lower exposure to risk factors, in view of remaining predominantly at home in safe surroundings.

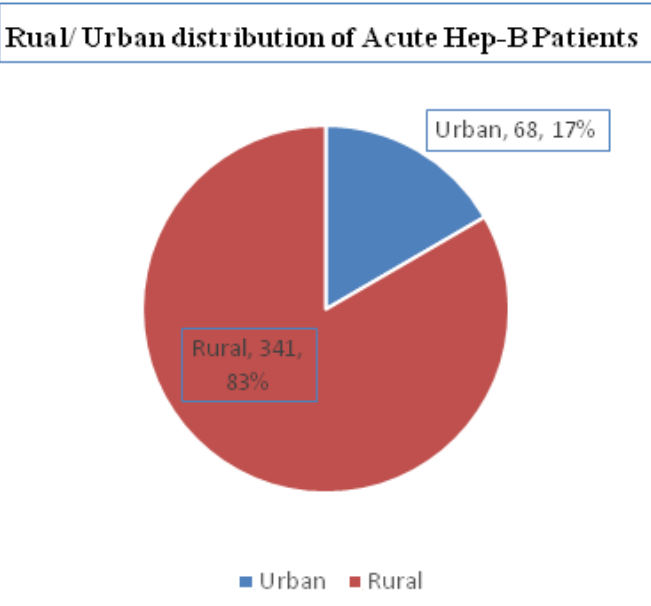

Figure I Rural and Urban distribution of Acute Hepatitis B Patients.

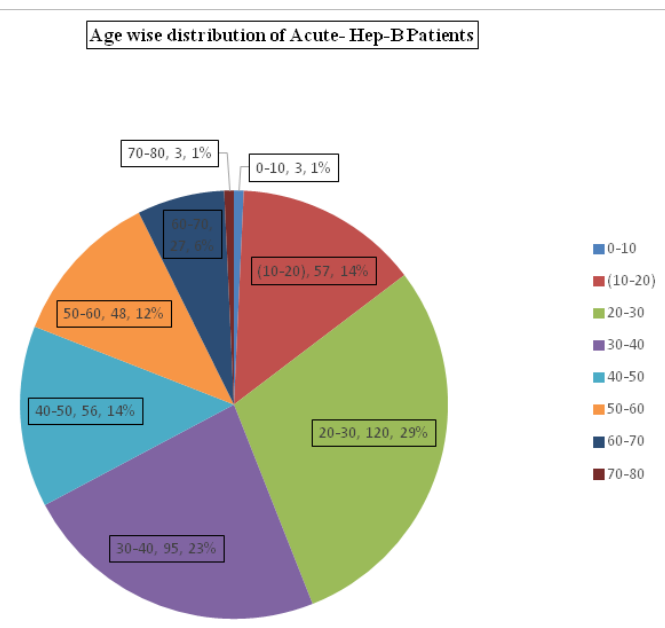

Figure 2 Age distribution of Acute Hepatitis B patients. 


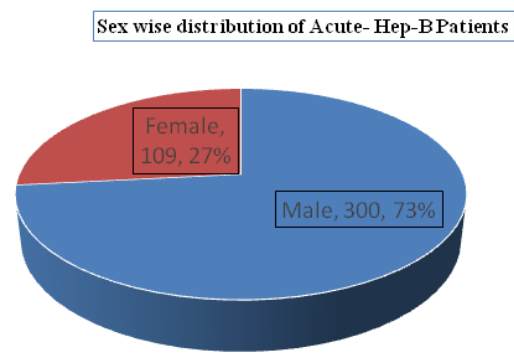

Figure 3 Sex distribution of Acute Hepatitis B patients.

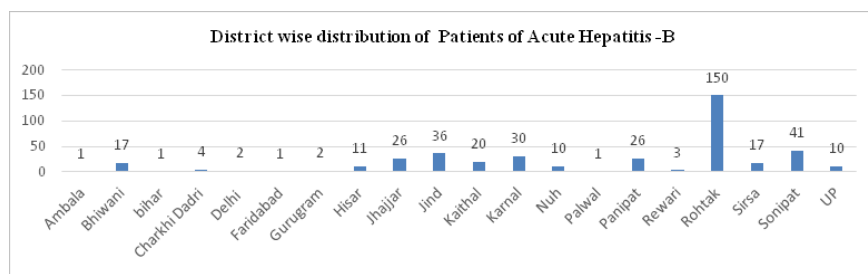

Figure 4 Geographical(district) wise distribution of Acute Hepatitis B patients.

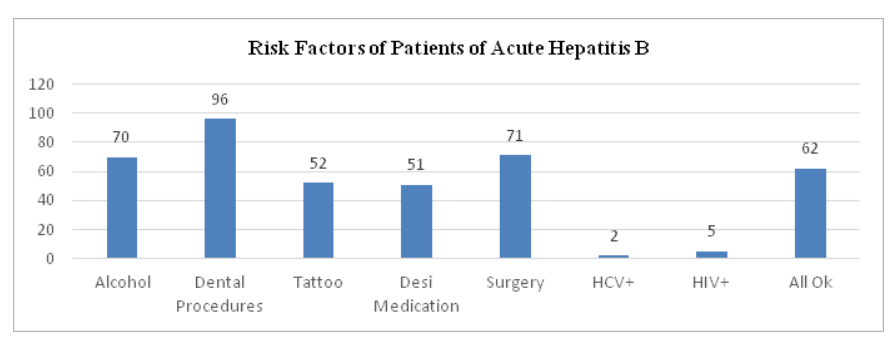

Figure 5 Risk Factors of Acute Hepatitis B patients.

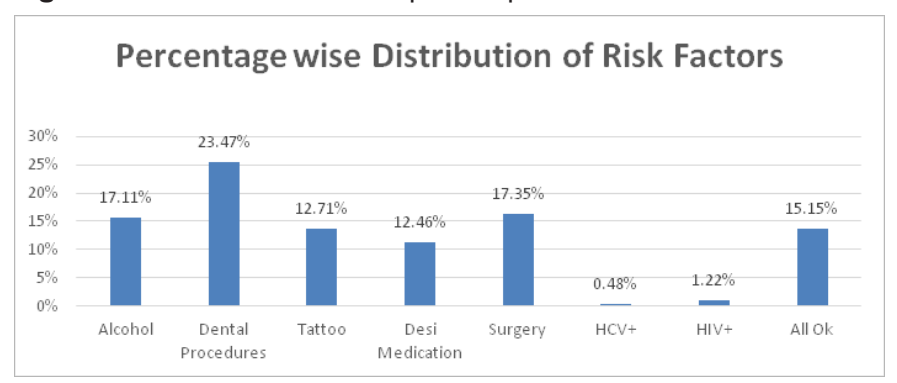

Figure 6 Percentage wise Distribution of Risk Factors in Acute Hepatitis B patients.

The accurate determination of incidence of HBV infection is not possible in view of large pool of asymptomatic cases and under reporting of cases. The reason for rural (village background) predominance of acute hepatitis B can be attributed mainly to unsafe needle practices. The other reasons attributed are percutaneous exposure to blood and blood products, tattooing, reuse of shaving blades by barbers previous history of surgery etc. The patients usually hide history of multiple sexual partners and intravenous drug abuse due to social stigma associated with them, thus leading to falsely low level of reporting of these risk factors. The epidemiological factors are different in developing and developed countries; hence strategies to control this disease should be planned accordingly. The most important intervention is to ensure safe blood donation as well as needle practices. The early detection of cases and timely treatment can also help in decreasing pool of this disease.

\section{Results}

The acute hepatitis B is becoming a significant health problem in certain hotspots, especially in young males of village background which can be due to unawareness, unhygienic practices and inadequate health care infrastructure. The unsafe needle injection practices are significant in spreading of this disease as evidenced by analysis of risk factors in this study like history of past surgical interventions, dental procedures and tattooing. It is important to increase the awareness level for this disease both in society and health professionals. In the initial stage, the thrust should be on hot spots having more disease burden.

\section{Conclusion}

Acute Hepatitis B is a significant problem and every patient presenting with jaundice must be screened for the same. All patients presenting with acute hepatitis B should be rigorously followed, as many of them, depending upon the age, can develop chronic hepatitis and ultimately chronic liver disease and hepatocellular carcinoma.

\section{Acknowledgments}

None.

\section{Conflicts of interest}

The authors declare that there are no conflicts of interest.

\section{Funding}

None.

\section{References}

1. Abraham P. Viral Hepatitis in India. Clin Lab Med. 2012;32(2):159-174.

2. Thyagarajan SP, Jayaram S, Mohanavalli B. Prevalence of HBV in general population in India. In: Sarin SK, Singal AK, Editors. Hepatitis B in India: problems and prevention. New Delhi: CBS; 1996. p. 5-16.

3. Dutta S. An overview of molecular epidemiology of hepatitis B virus (HBV) in India. Virol J. 2008;5:156.

4. Sarin SK, Dhiman RK, Eapen CA, et al. Technical Guidelines for Diagnosis \& management of Hepatitis 2019; National Viral Hepatitis Control Program, India.

5. Centers for Disease Control and Prevention. Table 3.2: clinical characteristics of reported cases of acute, symptomatic hepatitis B United States, 2012. USA: Centers for Disease Control and Prevention; 2012.

6. Hovi L, Saarinen UM, Jalanko H, et al. Characteristics and outcome of acute infection with hepatitis B virus in children with cancer. Pediatr Infect Dis J. 1991;10(11):809-812.

7. Walsh JH, Purcell RH, Morrow AG, et al. Post-transfusion hepatitis after open-heart operations. Incidence after the administration of blood from commercial and volunteer donor populations. JAMA. 1970;211(2):261265

8. Oliphant JW. Jaundice following Administration of human serum. Bull NY Acad Med. 1944;20:429-445.

9. Mortimer PP. Arsphenamine jaundice and the recognition of instrumentborne virus infection. Genitourin Med. 1995;71(12):109-119.

10. Centers for Disease Control and Prevention. 2012 National Notifiable Diseases and Conditions and Current case Definitions. USA: Centers for Disease Control and Prevention; 2012.

11. Parveen M, Vani M, Naveen M, et al. Epidemiological Profile of Hepatitis C Patients at India's New Hub - Haryana. Adv Res Gastroenterol Hepatol. 2015;1(1):555554. 
12. World Health Organization (WHO). Hepatitis B. World Health Organization Fact Sheet. Geneva: Swizerland; World Health Organization (WHO): 2020.

13. Lavanchy D. Hepatitis B virus epidemiology, disease burden, treatment, and current and emerging prevention and control measures. $J$ Viral Hepat. 2004;11(2):97-107.

14. Lok AS. Chronic hepatitis B. N Engl J Med. 2002;346(22):1682-1683.
15. Goldstein ST, Zhou F, Hadler SC, et al. A mathematical model to estimate global hepatitis B disease burden and vaccination impact. Int $J$ Epidemiol. 2005;34(6):1329-1339.

16. Te HS, Jensen DM. Epidemiology of hepatitis B and C viruses: a global overview. Clin Liver Dis. 2010;14(1):1-21. 